\title{
Cross-cultural and hemispheric laterality effects on the ensemble coding of emotion in facial crowds
}

\author{
Hee Yeon Im ${ }^{1,2}$, Sang Chul Chong ${ }^{3,4}$, Jisoo Sun ${ }^{3}$, Troy G. Steiner ${ }^{5}$, Daniel N. Albohn ${ }^{5}$, \\ Reginald B. Adams $\mathrm{Jr}^{5}$, and Kestutis Kveraga ${ }^{1,2}$ \\ ${ }^{1}$ Athinoula A. Martinos Center for Biomedical Imaging, Department Radiology, Massachusetts \\ General Hospital \\ 2Department of Radiology, Harvard Medical School \\ ${ }^{3}$ Graduate Program in Cognitive Science, Yonsei University \\ ${ }^{4}$ Department of Psychology, Yonsei University \\ ${ }^{5}$ Department of Psychology, The Pennsylvania State University
}

\begin{abstract}
In many social situations, we make a snap judgment about crowds of people relying on their overall mood (termed "crowd emotion"). Although reading crowd emotion is critical for interpersonal dynamics, the sociocultural aspects of this process have not been explored. The current study examined how culture modulates the processing of crowd emotion in Korean and American observers. Korean and American (non-East Asian) participants were briefly presented with two groups of faces that were individually varying in emotional expressions and asked to choose which group between the two they would rather avoid. We found that Korean participants were more accurate than American participants overall, in line with the framework on cultural viewpoints: Holistic versus analytic processing in East Asians versus Westerners. Moreover, we found a speed advantage for other-race crowds in both cultural groups. Finally, we found different hemispheric lateralization patterns: American participants were more accurate to perceive the facial crowd to be avoided when it was presented in the left visual field than the right visual field, indicating a right hemisphere advantage for processing crowd emotion of both European American and Korean facial crowds. However, Korean participants showed weak or nonexistent laterality effects, with a slight right hemisphere advantage for European American facial crowds and no advantage in perceiving Korean facial crowds. Instead, Korean participants showed positive emotion bias for own-race faces. This work suggests that culture plays a role in modulating our crowd emotion perception of groups of faces and responses to them.
\end{abstract}

Correspondence to: Kestutis Kveraga, A.A. Martinos Center for Biomedical Imaging, Department of Radiology, Harvard Medical School, Massachusetts General Hospital, Charlestown, MA 02129, kestas@nmr.mgh.harvard.edu.

Author Contributions

H. Y. Im, R. B. Adams, and K. Kveraga developed the study concept and all the authors contributed to study design. Testing and data collection were performed by J. Sun, T. G. Steiner, and D. N. Albohn. H. Y. Im analyzed the data and all the authors wrote the manuscript.

Declaration of Conflicting Interests

The authors declared that they had no conflicts of interest with respect to their authorship or the publication of the article. 


\section{Keywords}

culture; crowd emotion; ensemble coding; face perception; group perception

\section{Introduction}

We routinely encounter groups of people at work, school, or social gatherings. Efficiently reading the emotional states of crowds allows us to guide our own reactions and social behaviors. For example, rapidly inferring intent to commit violence from the facial expressions of a mob on the street can allow us to escape in time and avoid potential danger, perhaps by seeking help from another group that looks friendly. Likewise, reading the general mood and receptiveness of an audience allows us to adjust our ongoing behavior, by explaining in more detail or deferring on a point, for a more efficient communication. Such extraction of the prevailing crowd state can occur rapidly and efficiently, by representing the groups of faces as a higher-level description in the form of ensemble coding (Alvarez, 2011; Cohen, Dennett, \& Kanwisher, 2016; Haberman \& Whitney, 2012). Ensemble coding is the perception of the sum of parts rather than the perception of the individual parts within the sum; the perception of the sum is based on summary statistics (e.g., mean and/or variance) computed from multiple measurements of the parts, either by collapsing across the individual parts or across space and/or time. The key idea here is that the visual system exploits redundancies and regularities in scenes to extract ensemble representations from groups of similar items, without having to examine each individual object. Recent work on ensemble coding has demonstrated human observers' remarkable ability to extract average emotion (also termed "crowd emotion") from sets of faces (e.g., Elias, Dyer, \& Sweeny, 2016; Fischer \& Whitney, 2011; Haberman, Harp, \& Whitney, 2009; Haberman \& Whitney, 2007; Hubert-Wallander \& Boynton, 2015; Im et al., in press; Ji, Chen, \& Fu, 2014; Yang et al., 2013), facial identity (de Fockert \& Wolfenstein, 2009; Haberman \& Whitney, 2007; Leib et al., 2012; Leib et al., 2014; Neumann, Schweinberger, \& Burton, 2013), as well as a crowd's movements (Brunyé, Howe, \& Mahoney, 2014; Sweeny, Haroz, \& Whitney, 2012) and eye gaze direction (Florey et al., 2016; Sweeny \& Whitney, 2014).

Although perceiving average emotion of facial crowds is important in forecasting intentions of groups of people and governing observers' reactions towards them (e.g., which group would be safe to approach or better to avoid; Im et al., in press), little is known about sociocultural aspects of this process. To the best of our knowledge, only a single study has investigated the gender differences in recognizing average identity from a crowd of faces (Bai et al., 2015). It still remains unaddressed how different cultural groups (e.g., Westerners and Easterners) perceive crowd emotion when they see faces of same and different races (e.g., Caucasian and East Asian faces). The majority of previous studies on crowd emotion perception have exclusively employed photographs of Caucasian faces (Fischer \& Whitney, 2011; Haberman et al., 2009; Haberman \& Whitney, 2007; Hubert-Wallander \& Boynton, 2015; Im et al., in press). Although all these studies have been conducted in the Western countries, the specific breakdown of the participants' ethnic background was not available. We could find only a few exceptions that employed photographs of Asian faces (Chinese faces: Ji et al., 2014; Korean faces: Yang et al., 2013), but only when presented to a group of 
Chinese and Korean participants, respectively. Therefore, none of the existing studies on crowd emotion perception allows us to directly compare potential differences between Easterners and Westerners in extracting crowd emotion from groups of faces of Easterners vs. Westerners. A within-subject design in which participants view both facial crowds of Easterners and Westerners would be more effective than a between-subjects design because it would allow us to directly compare participants' performance for facial crowds of different races and reduce potential error variance associated with individual differences across groups. Characterizing differences in the perception of own-race-crowds vs. otherrace-crowds between Western participants vs. Eastern participants would represent an initial step in investigating the factors that mediate their different perceptual styles.

There is growing interest in empirically testing how differences in cultural experiences shape the way we perceive and respond to the visual world (for reviews, Han et al., 2012; Koelkebeck et al., 2017). Studies comparing Westerners and Easterners have provided convergent evidence for the different perceptual and cognitive styles of those populations (e.g., Lao, Vizioli, \& Caldara, 2013; Nisbett \& Miyamoto, 2005; Nisbett et al., 2001). For example, Westerners preferably focus on local information, whereas Easterners tend to show perceptual biases towards global information processing in objects (e.g., Masuda \& Nisbett, 2001), scene (e.g., Masuda \& Nisbett, 2006), and face perception (e.g., Blais et al., 2008; Caldara, Zhou, \& Miellet, 2010). Recent evidence also suggests that such cultural differences might rely on different tuning towards visual spatial frequency information across cultural groups (e.g., Miellet et al., 2013). For example, Westerners tend to use preferentially high spatial frequency information from foveal vision, whereas Easterners preferentially process contextual information by relying on extra-foveal vision for face recognition (Miellet et al., 2013), change detection of both low-level visual stimuli (e.g., color blocks; Boduroglu, Shah, \& Nisbett, 2009), and scene perception (Masuda \& Nisbett, 2001). The cultural difference in processing styles between Westerners allocating more attention to local and goal-directed objects and Easterners with broader or more holistic processing can emerge as early as 80 milliseconds after the onset of a visual stimulus, suggesting that such cultural difference is deeply ingrained into the early perceptual processing (Kitayama \& Murata, 2013; Lao et al., 2013).

The different processing styles between Westerners and Easterners also greatly influence how they process facial expressions. For example, Japanese participants are more likely to use overall resemblance rather than local feature matching when they identify a prototypic face for a set of discrete exemplars relative to Caucasian American participants (Miyamoto, Yoshikawa, \& Kitayama, 2011), and tend to look more at the surrounding people relative to Westerners (Masuda et al., 2008). Japanese participants' rating of emotion of a central person is more influenced by emotions of other surrounding faces (Ito, Masuda, \& Li, 2013; Russell et al., 2015), and also shows greater event-related brain potential (ERP) of N400 (negative-going deflection ERP around $400 \mathrm{~ms}$ after stimulus onset) and the late positive complex (LPC after $500 \mathrm{~ms}$ from stimulus onset; Russell et al., 2015). This effect is further shown to be generalizable even when photos of real faces were used, the saliency of the central person's emotion was attenuated, and when viewing time of face images was limited up to 10 seconds (Masuda et al., 2012). A recent study demonstrated that Japanese and Canadian parents' processing styles play a key role in transmitting such different processing 
styles to their children by maintaining their culturally shaped behaviors in front of their children (Lee et al., 2017). These findings together provide evidence for cultural variations in affective processing of single faces.

In the current study, we aimed to examine cultural differences in perceiving crowd emotion from groups of faces varying in emotional expression. We directly compared crowd emotion perception of Westerners (Caucasian and African-American participants) versus Easterners (Korean participants) when they were viewing facial crowds that contained European American faces versus Korean faces. To our knowledge, this is the first attempt to examine how Westerners and Easterners perceive crowds of people's faces from their own cultural or other cultural groups, broadly construed. Perceiving crowd emotion from groups of people is an important aspect of social cognition because it allows observers to evaluate a potential social threat from collective mood of the crowds (Phillips, Weisbuch, \& Ambady, 2014): Facial groups tend to present a greater threat and carry a higher emotional cost (Douilliez et al., 2011) and tend to be perceived as angrier, compared to individuals (Mihalache, Allen, \& Sweeny, 2017). Given the previous findings of different styles in affective processing (e.g., Ito et al., 2013; Masuda et al., 2008; Masuda et al., 2012; Miyamoto et al., 2011; Russell et al., 2015), we hypothesized that Easterners would more accurately extract global information from a group of different emotional faces. Furthermore, emotion expressions on same- vs. other race faces are found to be recognized more accurately (Adams et al., 2009; Elfenbein \& Ambady, 2002, 2003; Young \& Hugenberg, 2010) but require longer processing time (Caldara et al., 2004). The longer processing time is suggested to be the result of more semantic representations, more resource allocation, and more affective reactions to their ingroup members (Brewer, 1979; Caldara et al., 2004; Tajfel \& Turner, 1986). Therefore, we also expected to observe greater accuracy and longer response times for processing their own-race crowds' emotion vs. other-race crowds' emotion.

Face perception has great social utility because emotional expressions forecast behavioral intentions of expressers (Adams et al., 2006; Horstmann, 2003; Marsh, Ambady, \& Kleck, 2005), which govern observers' fundamental social motivations accordingly. From a perceiver's perspective, for example, an angry face elicits an avoidance reaction while a happy face elicits approach reaction (Adams et al., 2006; Marsh et al., 2005; Elliot, 1999). The current study sought to examine how human observers would make use of extracted crowd emotion for these socially relevant and critical decisions for survival, when they encounter their own race groups' faces vs. other racial groups' faces. Rather than just asking which group looks angrier, therefore, we asked the participants to choose as quickly and accurately as possible which crowd of faces they would rather avoid. Because participants had to choose one of the two facial crowds presented, their decisions required a relative decision between the two. This allowed us to create task settings that were more representative of the type of social appraisal that we are often involved in on a daily basis: e.g., avoiding a group of strangers who look relatively more violent or suspicious in the streets or any other public places. Furthermore, in many cases those expressions may not be extreme but rather subtle. On each trial, we presented two crowds of faces (each crowd comprised either 4 or 6 faces, as in the example shown in Figure 1C), with one crowd presented in the left, and the other crowd in the right, visual hemifield. Each of the faces in these facial crowds was created by morphing two extreme facial expressions (happy and 
angry) of the same individual so that they had different emotional intensities ranging from happy to angry. Such manipulation led participants to process and average all the individual members in a crowd to extract the overall emotion of the facial crowd, rather than choosing only one or two individuals.

\section{Experiment 1}

\section{Methods}

Participants-A total of 25 Korean undergraduate students from Yonsei University (Seoul, South Korea) and 29 U.S. native students from the Pennsylvania State University (State College, PA, USA) participated in Experiment 1. Two participants (one Korean and one American) were excluded from further analyses because they made too many late responses (e.g., RTs longer than $2.5 \mathrm{sec}$ ); three American participants were excluded because they did not complete the two experimental blocks; one American participant was excluded because of too fast responses (e.g., RTs shorter than $0.2 \mathrm{sec}$ ) and accuracy lower than chance (50\%). Therefore, we ended up with including 48 participants (24 Korean and 24 American). The number of participants included in the current study is sufficient to provide enough statistical power, given our original study on crowd emotion perception where we have shown robust and replicable effects from 20-21 participants in each of five successive experiments (Im et al., in press). Thirteen participants were female among 24 Korean participants (11 male) and 12 participants were female among 24 American participants (12 male). Among the 24 American participants, 19 were European American, two were Hispanic, and three were African American. The average age of Korean participants was 20.29 (s.d.: 1.73) years old and the average age of American participants was 18.75 (s.d.: 1.03) years old. Korean participants were slightly, but significantly older than American participants (mean age difference $=1.54, t(46)=3.75, p<0.001)$. Among 24 Korean participants, three were lefthanded and 20 were right-handed (one was unknown), and among 24 American participants, two were left-handed and 22 were right-handed. All the participants had normal color vision and normal or corrected-to-normal visual acuity. The informed written consents were obtained according to the procedures of the Institutional Review Board at Yonsei University and at the Pennsylvania State University for Korean and American participants, respectively. The participants received a course credit for participation in the study.

Apparatus and stimuli-Stimuli were generated with MATLAB and Psychophysics Toolbox (Brainard, 1997; Pelli, 1997). In each crowd stimulus, either 4 or 6 morphed faces were randomly positioned in each visual field (right and left) on a white background. Therefore, our facial crowd stimuli comprised either 8 or 12 faces. We used face-morphing software (Norrkross MorphX) to create a set of 51 morphed faces from two highly intense, prototypical facial expressions of the same person for a set of six different identities (3 Korean males and 3 European American males, shown in Figure 1A). The Korean face stimuli were taken from the Yonsei Face Database (Yang, Chung, \& Chong, 2015), and the White U.S. face stimuli (European American) were taken from the NimStim Emotional Face Stimuli database (Tottenham et al., 2009). The emotional expression of the faces ranged from 100\% happy and 100\% angry, with different proportions of morphing between these two (e.g., the neutral with morph of 50\% happy and 50\% angry faces). The morphed face 
images were linearly interpolated (in $2 \%$ increments) between two extreme faces. Such a morphing approach was adapted from the previous studies on ensemble coding of faces (e.g., Haberman \& Whitney, 2007). One of the two crowds in either left or right visual field was always neutral (on average), and the other had emotional crowds on average (e.g., very happy crowd: morphing of angry $32 \%$ and happy $68 \%$ ) somewhat happy: morphing of angry $40 \%$ and happy $60 \%$; somewhat angry: morphing of angry $60 \%$ and happy $40 \%$; and very angry: morphing of angry $68 \%$ and happy $32 \%$, see Figure 1B). To make an emotional crowd have the mean emotion of one of these morphing ratios, emotionally varying individual faces were randomly chosen from the continuum between $100 \%$ angry and $100 \%$ happy.

In order to avoid the possibility that participants simply sampled one or two single faces from each set and compare them to do the crowd emotion task, we ensured that $50 \%$ of the individual faces in the neutral set were more expressive than $50 \%$ of the individual faces in the emotional sets to be compared. For example, half of the members of the neutral set were angrier than a half of the members of the angry crowd. This manipulation allowed us to assess whether participants used such "sampling strategy" (Myczek \& Simons, 2008) rather than extracting the average crowd emotion because sampling one or two members in a set would only yield $50 \%$ of accuracy in this setting. On one half of the trials, the emotional stimulus (i.e., happy or angry) was presented in the left visual field (LVF) and the neutral stimulus was presented in the right visual field (RVF), and it was switched for the other half of the trials. Each face image subtended $2 \times 2$ of visual angle, and face images were randomly positioned within an invisible frame subtending $13.29 \times 18.29$, each in the left and right visual fields. The distance between the proximal edges of the invisible frames in left and right visual fields was 3.70. In order to test the visual hemifield effect, participants were asked to look at the center fixation before each trial began and keep their eyes fixated to the center during the stimulus presentation. By using the identical experimental settings including the size of the display, viewing distance, and the offset between the left and right visual fields, we have consistently shown the strong and reliable laterality effects on perception of crowd emotion (Im et al., in press).

Procedure-Figure 1C illustrates a sample trial. We ensured that both Korean and American participants performed the tasks in the same experimental settings and procedures. Participants sat in a chair at individual cubicles and viewed the visual stimuli for 1 second, which was followed by a blank screen for 1.5 seconds. The participants were instructed to make a key press as soon as possible to indicate which of the two facial crowds on the left or right they would rather avoid. They were explicitly informed that the correct answer was to choose to avoid the facial crowd showing a more negative (e.g., angrier) emotion on average. Responses that were made after 2.5 seconds were considered late and excluded from data analyses. Feedback for correct, incorrect, or late responses was provided after each response. The same written instructions were translated into Korean and English to be provided to Korean and American participants to emphasize that it is critical to make responses as quickly and as accurately as they could. The written instructions for Experiment 1 are provided both in English and Korean in Appendix 1. 
Korean face stimuli and European American face stimuli were presented in two separate blocks, in a counterbalanced order across participants. Each experimental block had a 4 (different angry/happy morphing ratios: angry $32 \%+$ happy 68\%, angry $40 \%+$ happy $60 \%$, angry $60 \%$ + happy $40 \%$, and angry $68 \%$ + happy $32 \%$ ) x 2 (visual field of presentation, LVF and RVF) x 2 (set size: 8 and 12 faces total) design, with 20 repetitions per condition. This yielded 320 trials total, and the sequence of the trials was randomized within each block.

\section{Results}

Consistent with our previous studies (e.g., Im et al., in press; Yang et al., 2013), we did not find any difference in accuracy (all $p s>0.551$ ) and RT (all $p s>0.773$ ) between different set sizes of the facial crowds ( 8 vs. 12 faces total; Figures 2A and 2B) in both cultural groups of participants, suggesting that extraction of crowd emotion does not require serial processing of each individual crowd member, but more likely occurs in parallel. Because there was no effect of crowd size, we collapsed the data from the different crowd size conditions for further analyses.

The overall accuracy for Korean and European American facial crowds was $64.57 \%$ (s.d.: 5.13) and $67.17 \%$ (s.d.: 5.83) in Korean participants, and $49.26 \%$ (s.d.: 6.93 ) and $59.23 \%$ (s.d.: 7.33) in American participants, respectively (Figure 2C). The accuracy of the Korean participants was higher than the chance level (50\%) for both the Korean facial crowds ( $t(23)$ $=13.922, p<0.001)$ and the European American facial crowds $(t(23)=14.443, p<0.001)$. The performance of the American participants was significantly higher than chance only for European American facial crowds $(t(23)=6.170, p<0.001)$, but not for Korean facial crowds $(t(23)=0.5245, p=0.605)$. Both Korean and American participants showed higher accuracy for European American facial crowds compared to Korean facial crowds, and Korean participants were more accurate than American participants both for Korean and European American facial crowds. Repeated-measures ANOVA with a within-subject factor of the race of facial crowds and a between-subject factor of the culture of participants (Western vs. Eastern) confirmed these observations with the significant main effects of the race of facial crowds $(F(1,46)=27.746, p<0.001)$ and the culture of the participants $(F(1,46)=69.34, p<0.001)$. The interaction between these factors was also significant $(F(1,46)=9.525, p<0.01)$. Specifically, the interaction showed that American participants were less accurate for Korean facial crowds compared to European American facial crowds, whereas Korean participants were equally accurate for both Korean and European American facial crowds. Furthermore, post hoc Tukey's HSD pairwise comparison tests revealed higher accuracy for European American facial crowds than for Korean facial crowds in American participants $(p<0.05)$ and higher accuracy in Korean participants than American participants, both for Korean and European American facial crowds $(p s<0.05)$.

The median response time (RT) of Korean participants was 1.120 seconds (s.d.: 0.237) for Korean facial crowds and 1.036 seconds (s.d.: 0.214) for European American facial crowds. The median RT for American participants was 0.806 seconds (s.d.: 0.255) for Korean facial crowds and 0.916 seconds (s.d.: 0.273) for European American facial crowds (Figure 2D). The main effect of the culture of the participants was significant $(F(1,46)=10.95, p<0.01)$, 
with Korean participants being overall slower than American participants. Although the main effect of the race of facial crowds was not significant $(F(1,46)=0.232, p=0.632)$, the interaction between the culture of the participants and the race of facial crowds was statistically significant $(F(1,84)=13.143, p<0.001)$. Specifically, the interaction showed that Korean participants made faster avoidance responses for European American facial crowds than for Korean facial crowds, whereas American participants made faster avoidance responses for Korean facial crowds than for European American facial crowds. Post hoc Tukey's HSD pairwise comparison tests confirmed significantly faster RTs for European American than Korean facial crowds in Korean participants $(p<0.04)$, and faster RTs for Korean than European American facial crowds in American participants $(p<0.05)$. Moreover, RT was significantly faster in American participants than Korean participants for both Korean and European American facial crowds $(p s<0.05)$.

Our recent work has shown the highly replicable effects of hemispheric asymmetry in reading crowd emotion (Im et al., in press). As in the current study, participants had to make a relative judgment on which one of the two crowds (either between an angry vs. a neutral crowd or between a neutral vs. a happy crowd) looked angrier and to be avoided. Participants' accuracy was greater when a facial crowd to be chosen for the avoidance task was presented in the left visual field (LVF) than in the right visual field (RVF), regardless of the actual emotional valence of the facial crowd (whether angry or neutral). Because in the previous study we only recruited American participants and presented them with facial crowds comprising faces from the Ekman face set (Ekman \& Friesen, 1976), it is not clear whether this right hemispheric advantage for processing the goal-relevant crowd emotion is universal across different cultures. Therefore, we next examined the patterns of laterality effects in Korean and American participants presented with new Korean and European American facial crowd stimuli.

As in the previous study (Im et al., in press), we found the right hemisphere advantage both for an angry crowd chosen over a neutral crowd and for a neutral crowd chosen over a happy crowd in American participants' responses to European American facial crowds. As shown in Figure 3A, the accuracy for an angrier crowd (averaged across the two different morphing ratios: angry $60 \%$ + happy $40 \%$ and angry $68 \%$ + happy $32 \%$ ) chosen over a neutral crowd (angry 50\% + happy 50\%) was greater when it was presented in the LVF. Similarly, the accuracy for the neutral crowd chosen over a happier crowd (averaged across the two different morphing ratios: angry $32 \%$ + happy $68 \%$ and angry $40 \%$ + happy $60 \%$ ) was also greater when it was presented in the LVF. Repeated-measures two-way ANOVA with factors of the visual field containing the facial crowd to be chosen (LVF and RVF) and the emotional valence of the crowd (angrier and neutral) confirmed the significant main effect of the visual field $(F(1,23)=14.424, p<0.001)$, specifically, accuracy for the LVF being greater than the RVF. The main effect of emotional valence was also significant $(F(1,23)=$ $5.749, p<0.03$ ), with the accuracy for the comparison between a neutral vs. a happy crowd being greater than the comparison between an angry vs. a neutral crowd. The interaction, however, was not significant $(F(1,23)=0.382, p=0.543)$. Post hoc Tukey's HSD pairwise comparison tests also revealed significantly higher accuracy for an angry crowd in the LVF than the RVF, and for a neutral crowd in the LVF than the RVF ( $p s<0.05)$. 
Likewise, we also found a significant main effect of the visual field with the Korean facial crowd stimuli $(F(1,23)=4.919, p<0.04$; Figure 3B), with the LVF being more accurate than the RVF. Neither the main effect of emotional valence $(F(1,23)=0.009, p=0.924)$ nor the interaction $(F(1,23)=0.006, p=0.980)$ was significant for the Korean facial crowd stimuli. Post hoc Tukey's HSD pairwise comparison tests further revealed significantly greater accuracy both for an angry crowd and for a neutral crowd in the LVF than the RVF ( $p s<$ 0.05 ). This is a direct replication of our previous study (Im et al., in press), in which we showed groups of Ekman faces (Ekman \& Friesen, 1976) to American undergraduate participants using a different set of white American faces. Furthermore, this result also shows that this laterality effect is still observed in American participants when they view a set of other-race facial stimuli (i.e., Korean faces).

We also examined the laterality effect in the Korean participants' responses to American and Korean facial crowds separately. For European American facial crowds (Figure 3C), we observed a similar, but weaker trend in the right hemisphere advantage for the crowd emotion to be chosen: The main effect of visual field was only marginally significant $(F(1,23)=3.045, p=0.094)$. The main effect of emotional valence was not significant $(F(1,23)=1.195, p=0.286)$, although the interaction was significant $(F(1,23)=4.351, p<$ 0.05). Post hoc Tukey's HSD pairwise comparison tests further revealed the nature of the interaction in which a neutral crowd of European American faces was perceived more accurately in the LVF than the RVF $(p<0.05)$.

For the crowds of Korean faces, we found that the main effect of visual field was not significant $(F(1,23)=0.080, p=0.780$; Figure 3D). Instead, there was a significant main effect of emotional valence $(F(1,23)=8.684, p<0.01)$, with the accuracy being greater when Korean participants had to choose a neutral over a happy crowd than when they had to choose an angry over a neutral crowd. The interaction was also significant $(F(1,23)=6.213$, $p<0.03)$. Post hoc Tukey's HSD pairwise comparison tests revealed significantly greater accuracy for an angry crowd in the RVF compared to the LVF ( $p<0.05$ ), but no significant difference for a neutral crowd in the LVF vs. RVF $(p=0.270)$. Together, American participants seemed to show more pronounced right hemisphere advantage for comparing the emotion of two facial crowds regardless of the race of facial crowds, compared to Korean participants who showed either weak or no right hemisphere advantage.

Previous studies have reported clear sex-related differences in perception of a single face (e.g., Rehnman \& Herlitz, 2006; McBain et al., 2009; Heisz et al., 2013) and crowds of faces (Bai et al., 2015). As a secondary interest, therefore, we examined whether there were any gender-related differences in accuracy for crowd emotion perception in Korean participants vs. in American participants. We conducted a three-way ANOVA with two between-subjects factors of Gender of participants (Female vs. Male) and Race of participants (Korean vs. American) and a within-subject factor of Race of facial crowds (Korean vs. American). We found the interaction between the two between-subjects factors (Gender of participants $\mathrm{x}$ Race of participants) was the only significant effect $(F(1,44)=6.747, p=0.013)$. Specifically, Korean male participants were more accurate than Korean female participants both for Korean and European American facial crowds (post-hoc $t$-test, Bonferroni-corrected $p s<0.03$ ), whereas there was no significant difference between the accuracy displayed by 
American male and female participants (post-hoc $t$-test, Bonferroni-corrected $p s>0.111$ ). Lack of accuracy differences between American male and female participants was also reported in previous work (Im et al., in press). Other effects (main effect of Gender of participants $F(1,44)=0.110, p=0.742)$, the interaction between the race of the stimulus and the gender of participants $(F(1,44)=0.095, p=0.760)$, the three-way interaction of the race of stimulus, race of participants, and the gender of participants was not significant, either $(F(1,44)=0.197, p=0.660)$.

\section{Experiment 2}

In the first experiment, we found differences between Korean vs. American participants in the overall accuracy, RT, and the laterality effects when they made avoidance decisions about emotional crowds of Korean and European American faces. In particular, we observed substantial differences in the overall accuracy and RT, even though we provided both groups of participants with the same instructions (translated into Korean and English) to emphasize both speed and accuracy of responses. Overall, Korean participants made much slower and more accurate responses, whereas American participants tended to make much faster but less accurate responses. In Experiment 2, we further tested if the differences found were due to a speed-accuracy trade-off between the Korean and American participants. In this follow-up study we employed instructions that differentially stressed speed for Korean participants and accuracy for American participants, respectively. If the difference in perception accuracy between the Korean and American participants was due to a speed-accuracy trade-off in these groups, stressing speed for Korean participants and stressing accuracy for American participants should reduce the group differences. However, if the differences in perception accuracy and speed we observed in Experiment 1 reflect something else (e.g., different perceptual and cognitive styles), rather than a mere speed-accuracy trade-off, we should still observe differences in accuracy and RT between Korean and American participants.

\section{Methods}

Participants-A new group of 46 undergraduate students from Yonsei University $(N=24)$ and from the Pennsylvania State University $(N=22)$ participated in Experiment 2 . All the participants had normal color vision and normal or corrected-to-normal visual acuity. One participant was excluded from further analyses because of incomplete experimental blocks. Therefore, we ended up including 45 participants (24 Korean and 21 American). Eleven participants were female among 24 Korean participants ( 13 male) and 10 participants were female among 21 American participants (11 male; 19 European American and 2 African American). The average age of Korean participants was 20.08 (s.d.: 2.30) years old and the average age of American participants was 18.86 (s.d.: 1.06) years old. Korean participants were slightly, but significantly older than American participants (mean age difference $=$ $1.22, t(43)=2.23, p<0.05)$. Among 24 Korean participants, two were left-handed and 20 were right-handed (two were unknown), and 22 American participants, one was left-handed and 21 were right-handed.

Apparatus and stimuli-All the aspects of the apparatus and the stimuli were identical to those in Experiment 1. 
Procedure-All the aspects of the experimental procedure were identical to those in Experiment 1, except that the written and verbal instructions provided to Korean and American participants were revised. For Korean participants, speed was particularly emphasized over accuracy whereas for American participants, accuracy was particularly emphasized over speed. The actual instructions given to Korean and American participants are provided in Appendix 2 both in Korean and English.

\section{Results}

Korean participants: stressing response speed-The median RTs in Experiment 2 were 0.944 seconds (s.d.: 0.178) for Korean facial crowds and 0.911 seconds (s.d.: 0.171) for European American facial crowds (Figure 2F). In order to test whether the different instruction stressing the response speed was effective, we compared the RTs obtained in Experiment 2 with those in Experiment 1. Repeated measures ANOVA with a withinsubjects factor of the race of facial crowds (European American faces vs. Korean faces) and a between-subjects factor (Experiment 1 vs. Experiment 2) revealed significantly faster RTs for Experiment 2 than Experiment $1(F(1,46)=7.340, p<0.01)$, and significantly faster RTs for European American facial crowds than Korean facial crowds $(F(1,46)=11.332, p<$ $0.01)$, although the interaction was not significant $(F(1,46)=2.161, p=0.148)$. Thus, our new instruction that emphasized the response speed was effective resulting in faster responses of Korean participants in Experiment 2.

Despite the faster RT's for Korean participants in Experiment 2, their accuracy did not decrease significantly (Figure 2E). Repeated-measures ANOVA showed that Korean participants' accuracies in Experiments 1 and 2 were not significantly different $(F(1,46)=$ $0.825, p=0.329$ ). Therefore, stressing response speed did not seem to impair Korean participants' response accuracy, but did result in faster RT's. We also found that the accuracy for European American facial crowds was significantly greater than for Korean facial crowds overall $(F(1,46)=29.258, p<0.001)$, replicating the previous study's results.

American participants: stressing response accuracy-In Experiment 1, we observed that American participants yielded much faster, but less accurate, responses compared to Korean participants. Thus, we asked a new group of American participants to try to spend enough time to view the stimuli and respond as accurately as possible in Experiment 2. However, the new instructions emphasizing accuracy over speed did not seem to improve American participants' accuracy. The accuracy was $52.71 \%$ (s.d.: 6.777) for Korean facial crowds and 53.44\% (s.d.: 7.319) for European American facial crowds (shown in Figure 2E). Repeated-measures ANOVA revealed that the accuracy in Experiment 2 was not significantly different from that in Experiment $1(F(1,43)=1.917, p=0.173)$, although the main effect of the race of facial crowds $(F(1,43)=14.02, p<0.001)$ and the interaction $(F(1,43)=10.46, p<0.01)$ were significant. We found a similar pattern for RT's of American participants in Experiment 1 vs. Experiment 2: The RT's for Experiment 1 were not significantly different from those for Experiment $2(F(1,43)=1.285, p=0.263)$; and the RTs for Korean facial crowds were faster than for European American facial crowds $(F(1,43)=4.318, p<0.05$; Figure $2 \mathrm{~F})$. Thus, we conclude that the instructions to American participants to focus on accuracy instead of speed failed to shift the participants' speed- 
accuracy trade-off criterion and did not significantly influence American participants' accuracy or RT. Comparing the accuracy and RT of Korean vs. American participants, post hoc Tukey's HSD pairwise comparison tests showed greater accuracy for the Korean participants than for the American participants both for Korean and European American facial crowds ( $p s<0.03$ ), but no difference in RT between Korean and American participants for Korean or European American facial crowds ( $p s>0.11$ ). We also found that the RT of Korean participants was faster for European American facial crowds than Korean facial crowds, whereas the RT of American participants was faster for Korean facial crowds than European American facial crowds (all ps $<0.05$ ), replicating the results of Experiment 1 .

Finally, we tested the laterality effects in making avoidance decision between two facial crowds when different instructions stressing either speed or accuracy were given to participants. As shown in Figures 4A and 4B, we found the same laterality effect when American participants viewed European American facial crowds and Korean facial crowds that we observed in Experiment 1. Both for American and Korean facial crowds, American participants in Experiment 2 were more accurate both for an angrier crowd and a neutral crowd when they were presented in the LVF than the RVF. This was confirmed by a repeated-measures ANOVA with significant main effect of visual field (European American faces: $F(1,20)=4.692, p<0.05$; Korean faces: $F(1,20)=4.370, p<0.05)$, and by significant Post hoc Tukey's HSD pairwise comparisons for an angry crowd in the LVF vs. RVF, and for a neutral crowd in the LVF vs. RVF ( $p s<0.05$ ). Unlike American participants, Korean participants showed weaker effects of the right hemisphere advantage. For European American facial crowds, the main effect of visual field was marginally significant $(F(1,23)=$ $3.250, p=0.08$; Figure 4C), with Korean participants' accuracy being greater for an angry crowd and a neutral crowd presented in the LVF than the RVF. Tukey's HSD pairwise comparisons showed that the accuracy was significantly greater for an angry crowd and a neutral crowd in the LVF than the RVF ( $p s<0.05$ ). For Korean facial crowds, Korean participants did not show a significant main effect of visual field $(F(1,23)=0.008, p=$ 0.931 ; Figure 4D), although the main effect of emotional valence was marginally significant $(F(1,23)=3.094, p=0.092)$, with the accuracy being greater when Korean participants chose a neutral over a happy crowd than when they chose an angry over a neutral crowd.

Finally, we examined the gender differences in Korean vs. American participants. We conducted a three-way ANOVA with two between-subjects factors of Gender of participants and Race of participants and a within-subject factor of the Race of facial crowds. Only the three-way interaction of Race of the facial crowd, Race of participants, and Gender of participants was significant $(F(1,41)=6.887, p=0.012)$. Specifically, we observed that American female participants had higher accuracy for Korean facial crowds than American male participants (post-hoc $t$-test, Bonferroni-corrected $p=0.051$ ). However, there was no difference in accuracy for European American facial crowds between American female vs. male participants (post-hoc $t$-test, Bonferroni-corrected $p=0.229$ ), again replicating our previous finding (Im et al., in press) from American female vs. male participants when they viewed only European American facial crowd stimuli. For Korean participants, no genderrelated differences reached significance. There was no main effect of Gender of participants $(F(1,41)=0.145, p=0.706)$, no interaction between Gender of participants and Race of 
stimulus $(F(1,41)=2.422, p=0.127)$, nor an interaction between Gender of participants and Race of participants $(F(1,41)=0.351, p=0.557)$.

\section{General Discussion}

The current study examined cultural differences between American and Korean participants in processing crowd emotion of faces belonging to either their own or another racial group. Here we report three main findings: 1) Korean participants were more accurate overall relative to American participants in choosing the angrier facial crowd to avoid, for both Korean and European American faces; 2) Korean participants made faster avoidance responses for European American facial crowds than for Korean facial crowds, whereas American participants made faster responses to Korean facial crowds than to European American facial crowds; 3) American participants showed highly lateralized response patterns in which they were significantly more accurate in choosing a facial crowd to avoid when it was presented in the left visual hemifield than in the right visual hemifield, indicating a right hemisphere advantage for processing crowd emotion. However, Korean participants showed weak to nonexistent laterality effects, with a slight right hemisphere advantage for European American facial crowds, and no right hemisphere advantage in perceiving Korean facial crowds.

The overall superior accuracy Korean participants displayed was not simply due to a speedaccuracy trade-off. Although Korean participants were much slower than American participants in Experiment 1, the instructions to Korean participants to focus on speed in Experiment 2 lowered their RTs to be on par with those of American participants, while their higher level of accuracy compared to American participants was maintained despite speedier responses. Conversely, American participants were less accurate than Korean participants in both Experiment 1 and 2, even when they were explicitly instructed to focus on making correct responses. One possibility is that the better accuracy in Korean participants compared to American participants for perceiving crowd emotion may be attributable to the higher sensitivity of global information extracted through holistic processing by Easterners compared to Westerns (Abel \& Hsu, 1949; Ji, Peng, \& Nisbett, 2000; Kitayama et al., 2003; Masuda \& Nisbett, 2001; Nisbett, 2003; Nisbett \& Miyamoto, 2005; Norenzayan \& Nisbett, 2000; Peng \& Nisbett, 1999). Such tendency can generalize to social contexts, suggesting that Westerners tend to see emotions in a group as a collection of individuals' feelings, whereas Easterners tend to see them as a group feeling. For example, Masuda et al. (2008) presented a set of cartoon pictures of an individual surrounded by a group of four other people and asked Japanese and Western participants to judge the emotion of the central person. They found that the emotion expressed by people surrounding the central person in the stimulus influenced judgment on the emotion of the central person in Japanese, but not in Western participants, and that Japanese participants looked at the people surrounding the central person more than Westerners did. Moreover, our finding is also in line with the previous studies that showed American participants perform better in the absolute task (e.g., reporting absolute length of a vertical line) whereas Japanese perform better in the relative task (e.g., reporting relative length proportion to the height of the surrounding frame around a vertical line; Hedden et al., 2008; Kitayama et al., 2003) and that Chinese participants are better at detecting co-occurrence and co-variation of events compared to American 
participants (Ji et al., 2000). Consistent with these previous findings, Korean participants in the current study showed a behavioral advantage on the global task in general, compared to American participants. However, the holistic processing pattern in Easterners can also be disruptive in some other cases when they have to process local features that are surrounded by interfering context or background (e.g., Masuda et al., 2008).

We found that both American and Korean participants made faster avoidance responses when they viewed facial crowds of other races (e.g., American participants being faster for Korean facial crowds and Korean participants being faster for European American facial crowds). Such a speed advantage for other-race faces has been previously observed in single face classification and recognition of human subjects (Caldara et al., 2004) and similar attentional bias toward conspecifics' emotions in primates (Kret et al., 2016). For example, by presenting Caucasian participants with single faces of Caucasians versus East Asians, Caldara et al., (2004) found that other-race faces (e.g., Asian faces) are classified faster than same-race faces (e.g., Caucasian faces). They suggested that typically lesser experience with other-race faces engenders fewer semantic representations, which in turn increases the speed of processing for such stimuli. Moreover, it has been suggested that people tend to be more favorable, in terms of affective reactions and resource allocation, to members of their own group (in-group) than to other groups (Brewer, 1979; Tajfel \& Turner, 1986). Allocating fewer resources to detailed features in out-group faces would also lead to insufficient individuation of members of the group, as it is known as the "out-group homogeneity effect" (Linville, Salovey, \& Fischer, 1986; Messick \& Mackie, 1989; Mullen \& Hu, 1989; Park, Judd, \& Ryan, 1991; Quattrone, 1986; Wilder, 1986). These factors could have contributed to our new findings of faster avoidance responses to facial crowds of other-race faces in both American and Korean participants. Therefore, our findings provide a conceptual replication and extension of theories of group perception, specifically defined by race, in more naturalistic social settings where observers perceive groups of emotional faces and make an affective evaluation between the groups.

Hemispheric asymmetry allows for processing of incoming perceptual inputs in parallel for competing goals (Rogers, Vallortigara, \& Andrew, 2013). Goal-dependent parallel processing is particularly useful when a large number of complex stimuli (such as a crowd of emotional faces) and competing cognitive goals tax the processing capacity of the visual system, as was the case in our task. For example, the right hemisphere (RH) is suggested to dominate in attending to novelty, detecting behaviorally goal-relevant, clear sensory events, executing rapid responses, and extracting global features, whereas the left hemisphere (LH) is thought to control responses for consideration of alternatives, to resolve ambiguities, and process local features by inhibiting RH (Corbetta \& Shulman, 2002; Rogers, 2002;

Robertson, Lamb, \& Knight, 1988). Recently, we have shown that the pattern of hemispheric lateralization in reading crowd emotion is dependent on the ongoing task goal in the context of social decisions (Im et al., in press). The right hemisphere advantage that was observed in Im et al. (in press) such that the facial crowd to be chosen (e.g., an angry crowd to be chosen over a neutral crowd and a neutral crowd to be chosen over a happy crowd during the avoidance) was perceived more accurately in the LVF than the RVF. Such an RH advantage is consistent with previous studies that have shown that the RH is dominant for global processing whereas the LH is dominant for local processing (e.g., Christie et al., 2012; Delis 
et al., 1986; Robertson et al., 1988; Robertson \& Ivry, 2000; Yovel et al., 2001). Because Im et al. (in press) presented American participants only with Caucasian faces selected from the Ekman face set (Ekman \& Friesen, 1976), it was unclear if this effect could be generalized to other cultural groups. The current study not only replicates their findings by using different stimuli (both for Caucasian and Korean facial crowds) in a different cohort of American participants, but also shows that Korean participants exhibit much weaker or minimal effects of the right hemisphere advantage compared to American participants. Instead of the right hemisphere advantage, Korean participants showed what may appear to be a positive emotion bias, with better accuracy for the comparison of a neutral vs. a happy crowd, but only for own-race faces (Korean facial crowds). Although this is a novel and interesting finding, it needs to be interpreted with caution. Because they performed a relative comparison task between two crowds (e.g., one neutral and the other happier), this effect could be the result of either a negative bias, if Korean participants were more likely to perceive a neutral crowd of Korean faces as being angrier, or a positive bias, if they were more likely to perceive a happy crowd of Korean faces as being happier. Future research efforts will be necessary to clarify this effect.

The current findings on differences in hemispheric lateralization between American participants (e.g., highly lateralized) and Korean participants (e.g., weakly or not lateralized) may reflect the different breadth of attention that results in different styles of engaging in the task and achieving the task goals. Where previous research has found that similar neural responses were recruited when processing same-culture facial stimuli (i.e., Japanese versus European Americans: Adams et al., 2010), the differences in laterality effects found here suggest distinct neural responses being recruited in participants from these cultures. This could be due to Eastern/Western differences in perceptual attention (see Park \& Kitayama, 2010). Westerners are suggested to be more narrowly focused, whereas Easterners have been suggested to have a broader focus, or be more holistic in the application of attention to perceptual objects (Nisbett et al., 2001). Kitayama \& Murata (2013) suggested that focused attention, more dominant in Westerners than Easterners, might be due to their strong independent orientation and corresponding tendency to pursue an action towards a goalrelevant object from the very beginning of processing (Kitayama \& Murata, 2013; Markus \& Kitayama, 1991; Varnum et al., 2010). Conversely, global attention for holistic processing, dominant in Easterners, might be due to their tendency to be receptive to a wider range of incoming information, which in turn allow them to adjust their behaviors accordingly (Kitayama \& Murata, 2013). Along with such qualitative differences in attentional breadth that results in different cognitive styles and strategies between Westerners and Easterners, the current study provides novel empirical evidence for cross-cultural variation in hemispheric lateralization closely associated with socio-affective decision-making in different cultural groups.

Previous studies have also reported qualitatively different patterns of hemispheric lateralization between Westerners and Easterners. To illustrate, Western subjects were better with stimuli presented in the left visual hemifield while Japanese subjects showed visual field symmetry in response to geometric shapes (Hatta \& Dimond, 1980); Westerners showed bilateral activity to face stimuli in the fusiform face area (FFA) whereas Easterners showed more right lateralization (Goh et al., 2010); and Westerners showed left hemisphere 
(LH) dominance whereas Easterners showed right hemisphere (RH) dominance in the baseline activation (Moss, Davidson, \& Saron, 1985) during the EEG resting state (with eyes-closed). Although the results are mixed, allowing for only limited conclusions, these findings along with our own findings suggest that there may be cross-cultural differences in hemispheric lateralization that reflect ethnic characteristics in behaviors, such as biases in perception, attention, cognition, and social attribution.

\section{Limitations and future research}

Before concluding, it is worth noting that our findings may be interpreted with respect to other hypotheses. Although our findings are consistent with the well-accepted framework on two distinct cognitive styles of information processing in Western and Eastern observers (analytical vs. holistic, respectively), several alternative explanations for our findings are also possible, due to a variety of practical constraints and confounding factors associated with cross-cultural experimental research. We wish to acknowledge three major alternative possibilities in interpreting our results.

First, it may be the case that Korean and American participants have used different implicit rules and criteria regarding the balance between speed and accuracy. Although we manipulated the test instructions and showed that the differences in accuracy and RT between Korean and American participants did not merely reflect differences in the speedaccuracy trade-off, we cannot completely rule out different tendencies in Korean and American participants to emphasize speed or accuracy, regardless of the instruction. American norms for timed measures typically remain within normal limits for a single error, suggesting a relatively specific speed-accuracy trade-off valued in American culture (Strutt et al., 2015). However, in other cultures speed and quality can be considered contradictory, and these cultures highlight the relative importance of accuracy over speed, for a good product is the result of a slow and careful process (Ardila, 2007). Our results may partly support this possibility in that Korean participants did not sacrifice their accuracy for speed when they were instructed to focus on making fast responses, while American participants did not slow down their responses when they were instructed to spend more time to be more accurate. Future work should specifically address such cross-cultural differences in speedaccuracy trade-off criteria to better understand how their influence on various perceptual and cognitive tasks.

Second, it is possible that the better overall accuracy in Korean participants than in American participants results from the type of the task we employed: avoiding one of the two emotional facial crowds. It has been shown that North Americans tend to be more attentive to approach-oriented information, whereas East Asians tend to be more attentive to avoidance-oriented information. For example, Hamamura et al. (2009) showed that American participants recalled more items describing approach-motivating events (e.g., gorgeous weather for hiking) whereas Japanese recalled more items describing avoidancemotivating events (e.g., stuck in a traffic jam). Moreover, it has been shown that Korean participants were more likely to embrace avoidance personal goals relative to European Americans (Elliot et al., 2001); Americans rated a tennis game that was framed as an opportunity to win as more important than one that was framed as an opportunity to avoid a 
loss, whereas Chinese participants showed the reverse pattern (Lee, Aaker, \& Gardner, 2000); and Canadian participants were motivated by success feedback more than failure feedback whereas Japanese participants were motivated by failure feedback than success feedback (Heine, Ide, \& Leung, 2001; Oishi \& Diener, 2003). Likewise, the better accuracy for Korean participants than Americans in the current study may be also associated with the avoidance task that we employed.

Finally, given that the face stimuli were morphed between extreme happy and extreme angry, it is possible that Korean participants are better at processing mixed emotions. It has been suggested that Easterners value and report experiencing more mixed emotion, for example, feeling happy and sad at the same time, compared to Westerners (Miyamoto, Ma, \& Wilken, 2017). Thus, it is possible that Korean participants could have been more accurate for processing each of the morphed faces in our facial crowds as well because of their better ability to read mixed emotion (e.g., subtle variation of emotions) from each morphed face as a result of mixture of happy and angry expressions. This is an interesting possibility that should be further investigated by future studies by comparing the ability of Easterners and Westerners to properly read complicated and mixed emotions from facial expressions. Moreover, it has been shown that Easterners rely on the eye region more, and on the mouth region less, than Westerners when they process facial expressions (Yuki, Maddux, \& Masuda, 2007). Thus, characterizing different patterns of eye movements across cultural groups during perception of crowd emotion of own-race vs. other race faces will be informative in understanding how Easterners and Westerners extract important social information to judge crowd emotion. In the current study, we were primarily interested in the comparison between different cultural groups (Korean vs. American) of participants to examine how sociocultural factors shape people's group perception. However, another potentially interesting avenue of research is exploring how participants' ethnicity interacts with cognitive aging and gender-related differences in perception of crowd emotion. Future cross-cultural studies of aging and gender effects will further investigate this with larger and more balanced sample size between age and gender groups.

\section{Conclusion}

In conclusion, the current study reports cross-cultural differences between American and Korean participants in reading crowd emotion in groups of faces. Cultural differences in group perception are a rarely investigated topic, although it seems that differences in social norms between different cultural and ethnic groups may be closely related to their interpersonal perception and interactions. Our work offers an initial inquiry into crosscultural differences in ensemble perception of faces with great social significance by presenting the same visual stimuli to two different cultural groups. Our work is an initial step to bridge a gap between the literature on ensemble coding of facial stimuli and a burgeoning area of cross-cultural work showing the importance of culture in understanding social perceptual processes. 


\section{Acknowledgments}

This work was supported by the National Institutes of Health R01MH101194 to K.K. and to R.B.A., Jr., and by the National Research Foundation of Korea (NRF) grant funded by the Korea government (NRF-2016R1A2B4016171) to S.C.C.

Data collection was conducted at the Pennsylvania State University for American participants and at Yonsei University for Korean participants. Informed written consent was obtained in all studies according to the procedures of the Institutional Review Board at the Pennsylvania State University and at Yonsei University. The participants received a course credit for their participation.

\section{References}

Abel TM, Hsu FI. Some aspects of personality of Chinese as revealed by the Rorschach Test. Journal of Projective Techniques. 1949; 13:285-301.

Adams RB Jr, Rule N, Franklin RG Jr, Wang E, Stevenson MT, Yoshikawa S, Nomura M, Sato W, Kveraga K, Ambady N. Cross-cultural reading the mind in the eyes: An fMRI investigation. Journal of Cognitive Neuroscience. 2010; 22:97-108. [PubMed: 19199419]

Alvarez GA. Representing multiple objects as an ensemble enhances visual cognition. Trends in Cognitive Sciences. 2011; 15(3):122-131. [PubMed: 21292539]

Ardila A. The impact of culture on neuropsychological test performance. International handbook of cross-cultural neuropsychology. 2007:23-44.

Bai Y, Leib AY, Puri AM, Whitney D, Peng K. Gender differences in crowd perception. Frontiers in Psychology. 2015; 6:1-12. [PubMed: 25688217]

Blais C, Jack RE, Scheepers C, Fiset D, Caldara R. Culture shapes how we look at faces. PLoS ONE. 2008; 3(8):e3022. [PubMed: 18714387]

Boduroglu A, Shah P, Nisbett RE. Cultural Differences in Allocation of Attention in Visual Information Processing. Journal of Cross-Cultural Psychology. 2009; 40(3):349-360. [PubMed: 20234851]

Brainard DH. The Psychophysics Toolbox. Spatial Vision. 1997; 10(4):433-436. [PubMed: 9176952]

Brewer MB. In-group bias in the minimal intergroup situation: A cognitive-motivational analysis. Psychological Bulletin. 1979; 86(2):307-324.

Brunyé TT, Howe JL, Mahoney CR. Seeing the crowd for the bomber: Spontaneous threat perception from static and randomly moving crowd simulations. Journal of Experimental Psychology: Applied. 2014; 20(4):303-322. [PubMed: 25347410]

Caldara R, Zhou X, Miellet S. Putting culture under the "Spotlight" reveals universal information use for face recognition. PLoS ONE. 2010; 5(3):e9708. [PubMed: 20305776]

Caldara R, Rossion B, Bovet P, Hauert CA. Event-related potentials and time course of the "otherrace" face classifcation advantage. Neuroreport. 2004; 15(3):487-491. [PubMed: 15094509]

Christie J, Ginsberg JP, Steedman J, Fridricksson J, Bonilha L, Rorden C. Global versus local processing: seeing the left side of the forest and the right side of the trees. Frontiers in Human Neuroscience. 2012:22. [PubMed: 22375111]

Cohen MA, Dennett DC, Kanwisher N. What is the Bandwidth of Perceptual Experience ? Trends in Cognitive Sciences. 2016; 20(5):324-335. [PubMed: 27105668]

Corbetta M, Shulman GL. Control of Goal-Directed and Stimulus-Driven Attention in the Brain. Nature Reviews Neuroscience. 2002; 3(3):215-229.

de Fockert J, Wolfenstein C. Rapid extraction of mean identity from sets of faces. Quarterly Journal of Experimental Psychology. 2009; 62(9):1716-1722.

Delis DC, Robertson LC, Efron R. Hemispheric specialization of memory for visual hierarchical stimuli. Neuropsychologia. 1986; 24:205-214. [PubMed: 3714025]

Ekman, P., Friesen, WV. Pictures of facial affect. Consulting Psychologists Press; Palo Alto, CA: 1976.

Elias E, Dyer M, Sweeny TD. Ensemble perception of dynamic emotional groups. Psychological Science. 2016; 28:193-203. [PubMed: 28036236] 
Elliot AJ, Chirkov VI, Kim Y, Sheldon KM. A cross-cultural analysis of avoidance (relative to approach) personal goals. Psychological Science. 2001; 12(6):505-510. [PubMed: 11760139]

Fischer J, Whitney D. Object-level visual information gets through the bottleneck of crowding. Journal of Neurophysiology. 2011; 106(3):1389-1398. [PubMed: 21676930]

Florey J, Clifford CWG, Dakin S, Mareschal I. Spatial limitations in averaging social cues. Scientific Reports. 2016; 6:32210. [PubMed: 27573589]

Goh JOS, Leshikar ED, Sutton BP, Tan JC, Sim SKY, Hebrank AC, Park DC. Culture differences in neural processing of faces and houses in the ventral visual cortex. Social Cognitive and Affective Neuroscience. 2010; 5:227-235. [PubMed: 20558408]

Haberman J, Harp T, Whitney D. Averaging facial expression over time. Journal of Vision. 2009; 9:113.

Haberman J, Whitney D. Rapid extraction of mean emotion and gender from sets of faces. Current Biology. 2007; 17:751-753.

Haberman, J., Whitney, D. Ensemble Perception: Summarizing the scene and broadening the limits of visual processing. In: Wolfe, J., Robertson, L., editors. From Perception to Consciousness: Searching with Anne Treisman. Oxford University Press; 2012.

Hamamura T, Meijer Z, Heine SJ, Kamaya K, Hori I. Approach-avoidance motivation and information processing: A cross-cultural analysis. Personality and Social Psychology Bulletin. 2009; 35(4): 454-462. [PubMed: 19164704]

Han S, Northoff G, Vogeley K, Wexler BE, Kitayama S, Varnum MEWW. A Cultural Neuroscience Approach to the Biosocial Nature of the Human Brain. Annual Review of Psychology. 2012; 64:335-359.

Hatta T, Dimond SJ. Comparison of Lateral Differences for Digit and Random Form Recognition in Japanese and Westerners. Perception. 1980; 6(2):368-374.

Hedden T, Ketay S, Aron A, Markus HR, Gabrieli JDE. Cultural influences on neural substrates of attentional control. Psychological Science. 2008; 19(1):12-17. [PubMed: 18181784]

Heine SJ, Ide E, Leung C. Divergent Consequences of Success and Failure in Japan and North America : An Investigation of Self-Improving Motivations and Malleable Selves. 2001; 81(4):599_ 615.

Hubert-Wallander B, Boynton GM. Not all summary statistics are made equal : Evidence from extracting summaries across time. Journal of Vision. 2015; 15:1-12.

Im HY, Albohn DN, Steiner TG, Cushing CA, Adams RB Jr, Kveraga K. Ensemble coding of crowd emotion: Differential hemispheric and visual stream contributions. Nature Human Behaviour. (in press).

Ji, L., Chen, W., Fu, X. Different Roles of Foveal and Extrafoveal Vision in Ensemble Representation for Facial Expressions. In: Harris, D., editor. Engineering Psychology and Cognitive Ergonomics. EPCE 2014. Lecture Notes in Computer Science. Vol. 8532. Springer; Cham: 2014.

Ji LJ, Peng K, Nisbett RE. Culture, control, and perception of relationships in the environment. Journal of Personality and Social Psychologycial Psychology. 2000; 78(5):943-955.

Kitayama S, Duffy S, Kawamura T, Larsen JT. Perceiving an Object and Its Context in Different Cultures: A Cultural Look at New Look. Psychological Science. 2003; 14(3):201-206. [PubMed: 12741741]

Kitayama S, Murata A. Culture modulates perceptual atention: An event-related potential study. Social Cognition. 2013; 31(6):758-769.

Kitayama S, Uskul AK. Culture, mind, and the brain: Current evidence and future directions. Annual Review of Psychology. 2011; 62:419-449.

Koelkebeck K, Uwatoko T, Tanaka J, Kret ME. How culture shapes social cognition deficits in mental disorders: A review. Social Neuroscience. 2017; 12:102-112. [PubMed: 26899265]

Kret ME, Jaasma L, Bionda T, Wijnen JG. Bonobos (Pan Paniscus) show an attentional bias toward conspecifics' emotions. Proceedings of the National Academy of Sciences of the United States of America. 2016; 113:3761-3766. [PubMed: 26976586]

Lao J, Vizioli L, Caldara R. Culture modulates the temporal dynamics of global/local processing. Culture and Brain. 2013; 1:158-174. 
Lee AY, Aaker JL, Gardner WL. The pleasures and pains of distinct self-construals: The role of interdependence in regulatory focus. Journal of Personality and Social Psychology. 2000; 78(6): 1122-1134. [PubMed: 10870913]

Leib AY, Fischer J, Robertson L, Whitney D. Ensemble crowd perception : A viewpoint-invariant mechanism to represent average crowd identity. Journal of Vision. 2014; 14:1-13.

Leib AY, Puri AM, Fischer J, Bentin S, Whitney D, Robertson L. Crowd perception in prosopagnosia. Neuropsychologia. 2012; 50(7):1698-1707. [PubMed: 22521873]

Linville PW, Salovey P, Fischer GW. Stereotyping and perceived distributions of social characteristics: An application to ingroup-outgroup perception. Prejudice, Discrimination and Racism. 1986:165208.

Markus HR, Kitayama S. Culture and the self: Implications for cognition, emotion, and motivation. Psychological Review. 1991; 98(2):224-253.

Masuda T, Ellsworth PC, Mesquita B, Leu J, Tanida S, Van De Veerdonk E. Placing the face in context: Cultural differences in the perception of facial emotion. Journal of Personality and Social Psychology. 2008; 94(3):94365-94381.

Masuda T, Nisbett RE. Attending holistically versus analytically: comparing the context sensitivity of Japanese and Americans. Journal of Personality and Social Psychology. 2001; 81(5):922-934. [PubMed: 11708567]

Masuda T, Nisbett RE. Culture and change blindness. Cognitive Science. 2006; 30:381-399. [PubMed: 21702819]

Messick DM, Mackie DM. Intergroup relations. Annual Review of Psychology. 1989; 40:45-81.

Miellet S, Vizioli L, He L, Zhou X, Caldara R. Mapping Face Recognition Information Use across Cultures. Frontiers in Psychology. 2013; 4:34. [PubMed: 23430143]

Moss EM, Davidson RJ, Saron C. Cross-cultural differences in hemisphericity: EEG asymmetry discriminates between Japanese and Westerners. Neuropsychologia. 1985; 23(1):131-135. [PubMed: 3974852]

Mullen B, Hu LT. Perceptions of Ingroup and Outgroup Variability: A Meta-Analytic Integration. Basic and Applied Social Psychology. 1989; 10(3):233-252.

Myczek K, Simons DJ. Better than average: Alternatives to statistical summary representations for rapid judgments of average size. Perception \& Psychophysics. 2008; 70(5):772-788. [PubMed: 18613626]

Neumann MF, Schweinberger SR, Burton AM. Viewers extract mean and individual identity from sets of famous faces. Cognition. 2013; 128(1):56-63. [PubMed: 23587844]

Nisbett, RE. The geography of thought: How Asians and West- erners think differently and why. New York: Free Press; 2003.

Nisbett RE, Miyamoto Y. The influence of culture: Holistic versus analytic perception. Trends in Cognitive Sciences. 2005; 9:467-473. [PubMed: 16129648]

Nisbett RE, Peng K, Choi I, Norenzayan A. Culture and systems of thought: holistic versus analytic cognition. Psychological Review. 2001; 108(2):291-310. [PubMed: 11381831]

Norenzayan A, Nisbett RE. Culture and causal cognition. Current Directions in Psychological Science. 2000; 9(4):132-135.

Oishi S, Diener E. Culture and well-being: The cycle of action, evaluation, and decision. Personality and Social Psychology Bulletin. 2003; 29:939-949. [PubMed: 15189614]

Park B, Judd CM, Ryan CS. Social Categorization and the Representation of Variability Information. European Review of Social Psychology. 1991; 2:211-245.

Park, H., Kitayama, S. Perceiving through culture: The socialized attention hypothesis. In: Adams, RB., JrAmbady, N.Nakayama, K., Shimojo, S., editors. The Science of Social Vision. Oxford University Press; 2010.

Pelli DG. The VideoToolbox software for visual psychophysics: transforming numbers into movies. Spatial Vision. 1997; 10(4):437-442. [PubMed: 9176953]

Peng K, Nisbett RE. Culture, dialectics, and reasoning about contradiction. American Psychologist. 1999; 54(9):741-754. 
Quattrone, GA. On the perception of a group's variability. In: Worchel, S., Austin, W., editors. The psychology of intergroup relations. 2. Chicago: Nelson-Hall; 1986. p. 25-48.

Robertson LC, Ivry R. Hemispheric asymmetry: attention to visual and auditory primitives. Current Directions in Psychological Science. 2000; 9:59-63.

Robertson LC, Lamb MR, Knight RT. Effects of lesions of temporal-parietal junction on perceptual and attentional processing in humans. The Journal of Neuroscience : The Official Journal of the Society for Neuroscience. 1988; 8(10):3757-3769. [PubMed: 3193178]

Rogers, LJ., Andrew, RJ. Comparative vertebrate lateralization. Cambridge University Press; 2002.

Rogers, LJ., Vallortigara, G., Andrew, RJ. Divided brains: The biology and behavior of brain asymmetries. Cambridge, UK: Cambridge University Press; 2013.

Sweeny TD, Haroz S, Whitney D. Perceiving Group Behavior: Sensitive Ensemble Coding Mechanisms for Biological Motion of Human Crowds. Journal of Experimental Psychology: Human Perception and Performance. 2012; 39(2):329-337. [PubMed: 22708744]

Sweeny TD, Whitney D. Perceiving Crowd Attention: Ensemble Perception of a Crowd's Gaze. Psychological Science. 2014; 25(10):1903-1913. [PubMed: 25125428]

Tajfel H, Turner JC. The social identity theory of intergroup behavior. Psychology of Intergroup Relations. 1986:7-24.

Tottenham N, Tanaka JW, Leon AC, McCarry T, Nurse M, Hare Ta, ... Nelson C. The NimStim set of facial expressions: Judgements from untrained research participants. Psychiatry Research. 2009; 168(3):242-249. [PubMed: 19564050]

Varnum MEW, Grossmann I, Kitayama S, Nisbett RE. Current Directions in Psychological Science The Origin of Cultural Differences in Cognition. Current Directions in Psychological Science. 2010; 19(1):9-13. [PubMed: 20234850]

Yang, J-W., Chung, K., Chong, SC. The Yonsei Face Database. Institute of Cognitive Science, Yonsei University; Seoul, Korea: 2015.

Yang J-W, Yoon KL, Chong SC, Oh KJ. Accurate but pathological: Social anxiety and ensemble coding of emotion. Cognitive Therapy and Research. 2013; 37:572-578.

Varnum ME, Grossmann I, Kitayama S, Nisbett RE. The origin of cultural differences in cognition: Evidence for the social orientation hypothesis. Current Directions in Psychological Science. 2010; 19(1):9-13. [PubMed: 20234850]

Wilder, DA. Social categorization: Implications for creation and reduction of intergroup conflict. In: Berkowitz, L., editor. Advances in experimental social psychology. Vol. 19. San Diego, CA: Academic Press; 1986. p. 293-355.

Yovel G, Levy J, Yovel I. Hemispheric asymmetries for global and local visual perception: effects of stimulus and task factors. Journal of Experimental Psychology: Human Perception and Performance. 2001; 27:1369-1385. [PubMed: 11766931]

\section{Appendix 1}

\section{Instruction for Experiment 1}

\section{a. Instruction written in Korean (for Korean participants)}

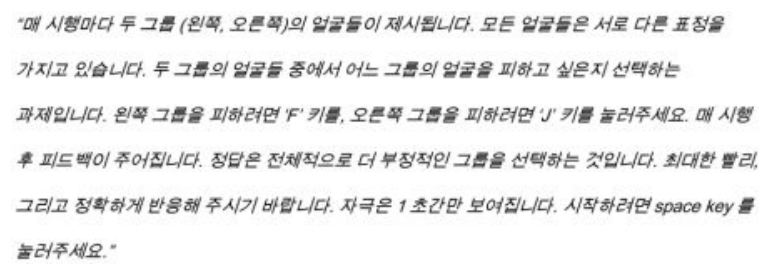




\section{b. Instruction written in English (for American participants)}

"In this experiment, you will be presented with two crowds of faces with varying emotional expressions. Your task is to choose which of the two groups of people (on the left or right) you would rather avoid. Press 'F' for left group, and press 'J' for right group on the display. The stimulus image will be presented very briefly. Please try to make a response as soon as possible. Also, please try to remain as accurate as possible. If you are ready to start, press a space key."

\section{Appendix 2}

\section{Instruction for Experiment 2}

\section{a. Instruction for Korean participants}

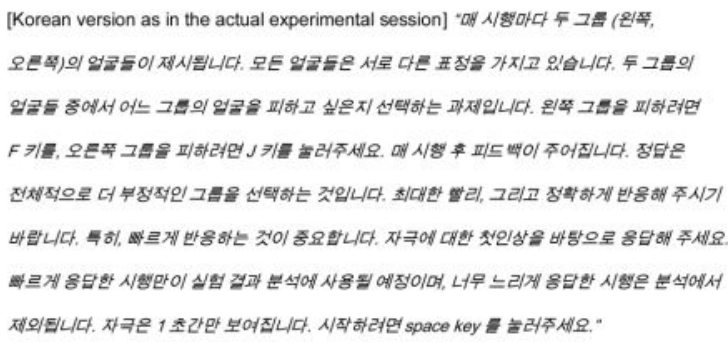

[English version, translated from Korean] "On each trial, two crowds of faces with varying emotional expressions will be presented for 1 second. Your task is to choose which of the two groups of people (on the left or right) you would rather avoid. Press ' $F$ ' to avoid left group, and press 'J' to avoid right group on the screen. Correct answer is to choose angrier (more negative) crowd on average. Feedback will be provided on each trial. Please try to remain as accurate as possible. However, we require fast responses for our analyses: Accuracy at the expense of speed will undermine our study, because responses that are too slow cannot be analyzed. Please rely on your first impression of the stimuli and try to make response as quickly as you can."

\section{b. Instruction for American participants}

On each trial, two crowds of faces with varying emotional expressions will be presented for 1 second. Your task is to choose which of the two groups of people (on the left or right) you would rather avoid. Press ' $F$ ' to avoid left group, and press ' $\mathrm{J}$ ' to avoid right group on the screen. Correct answer is to choose angrier (more negative) crowd on average. Feedback will be provided on each trial. Please try to remain as fast as possible. However, we require accurate responses for our analyses: Speed at the expense of accuracy will undermine our study, because only accurate responses can be analyzed. Please try to make response as accurately as you can. 
A

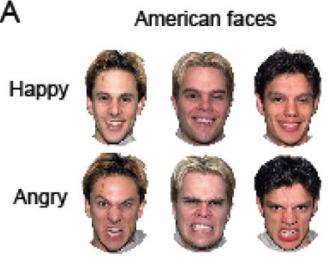

C

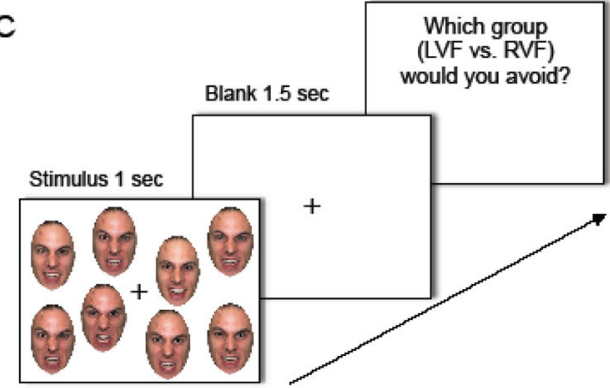

B

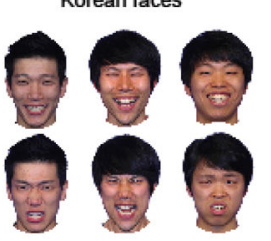

(1)

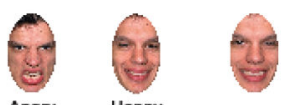

${ }_{32 \%}^{\text {Angry }}+\underset{68 \%}{\text { Happy }}=$ Very happy

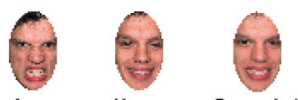

$\underset{40 \%}{\text { Angry }}+\underset{60 \%}{\text { Happy }}=\underset{\text { happy }}{\text { Somewhat }}$
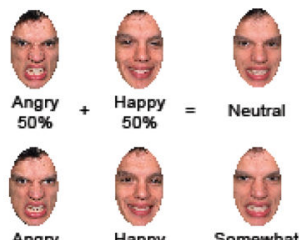

${ }_{60 \%}^{\text {Angry }}+{ }_{40 \%}^{\text {Happy }}=$ Somewhat

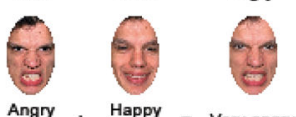

$\underset{68 \%}{\text { Angry }}+\underset{32 \%}{\text { Happy }}=$ Very angry

Figure 1. Samples stimuli and a sample experimental trial

(A) Happy and angry faces of three European American faces and Korean faces. (B) Example of different proportions of morphing of the happy and angry faces shown in Figure 1A. Morphing was done for each facial identity. (C) A sample trial. 
A

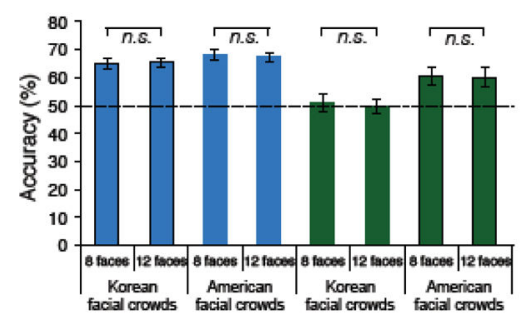

$\mathrm{C}$

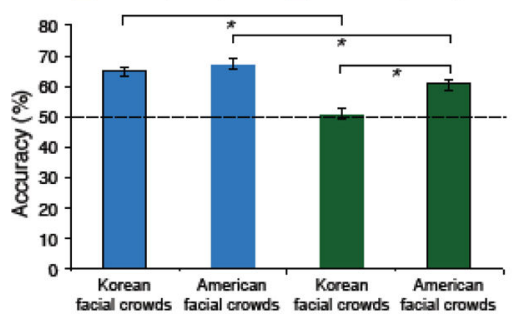

E

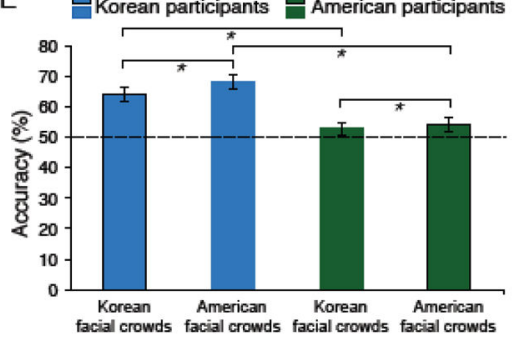

믈orean participants 믈 American participants

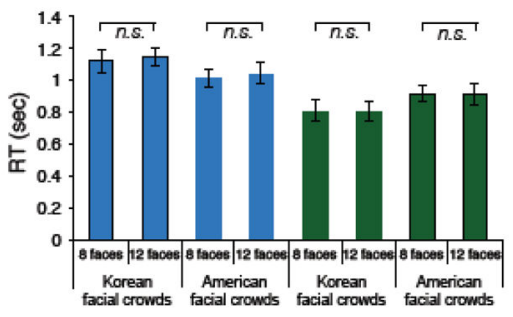

D $\square_{\text {Korean participants }}$ American participants

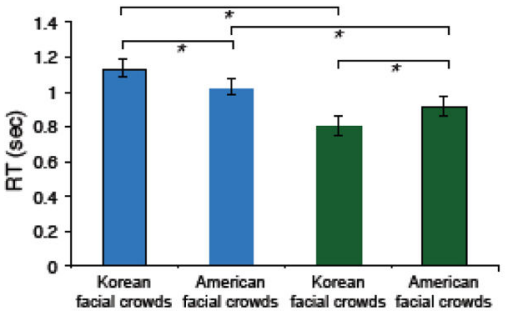

F

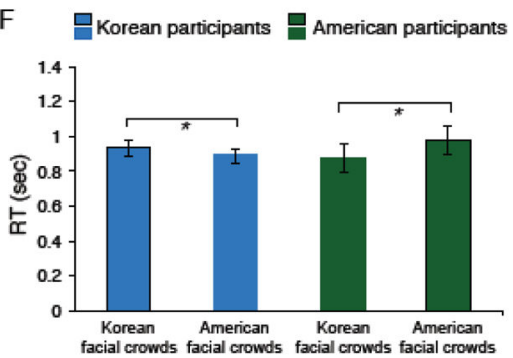

Figure 2. The main results in Experiment 1 (A-D) and in Experiment 2 (E-F)

(A) The set size effect on the overall accuracy. The blue bars indicate the accuracy in Korean participants and the green bars indicate the accuracy in American participants. The black outline indicates facial crowds of their own-race for Korean and American participants. The broken line indicates the chance level, 50\%. The error bars indicate the standard error of the mean (SEM). (B) The set size effect on the overall RT. (C) Experiment 1: The overall accuracy for Korean and American facial crowds in Korean participants (blue bars) and in American participants (green bars). (D) Experiment 1: The overall RT for Korean and American facial crowds in Korean participants (blue bars) and in American participants (green bars). (E) Experiment 2: The overall accuracy for Korean and American facial crowds in Korean participants (blue bars) and in American participants (green bars). (F) Experiment 2: The overall RT for Korean and American facial crowds in Korean participants (blue bars) and in American participants (green bars). 

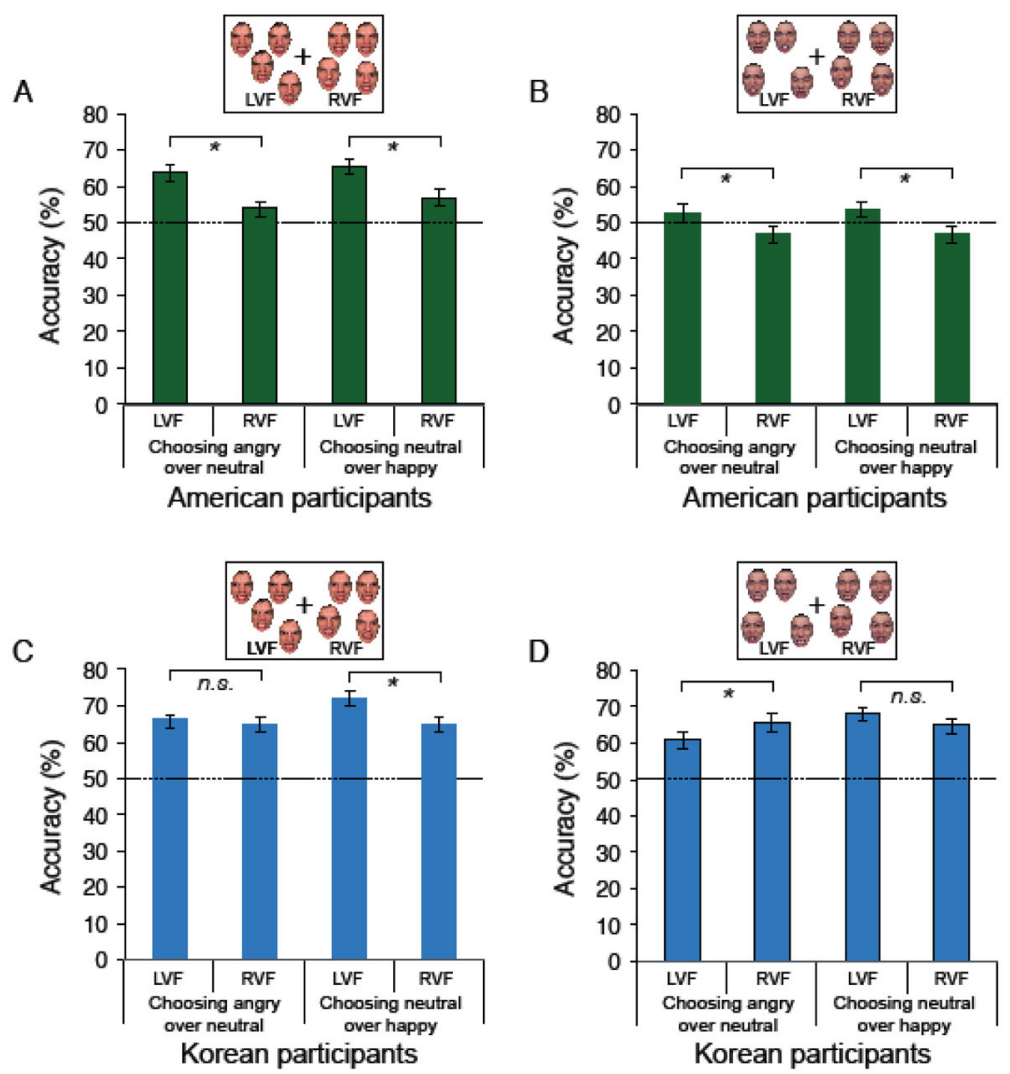

Figure 3. Laterality effect in Experiment 1

(A) The American participants' accuracy for American facial crowds when they had to choose an angrier and a neutral facial crowd presented in the LVF and the RVF. The broken line indicates the chance level, 50\%. The error bars indicate the standard error of the mean (SEM). (B) The American participants' accuracy for Korean facial crowds when they had to choose an angrier and a neutral facial crowd presented in the LVF and the RVF. (C) The Korean participants' accuracy for American facial crowds when they had to choose an angrier and a neutral facial crowd presented in the LVF and the RVF. (D) The Korean participants' accuracy for Korean facial crowds when they had to choose an angrier and a neutral facial crowd presented in the LVF and the RVF. 

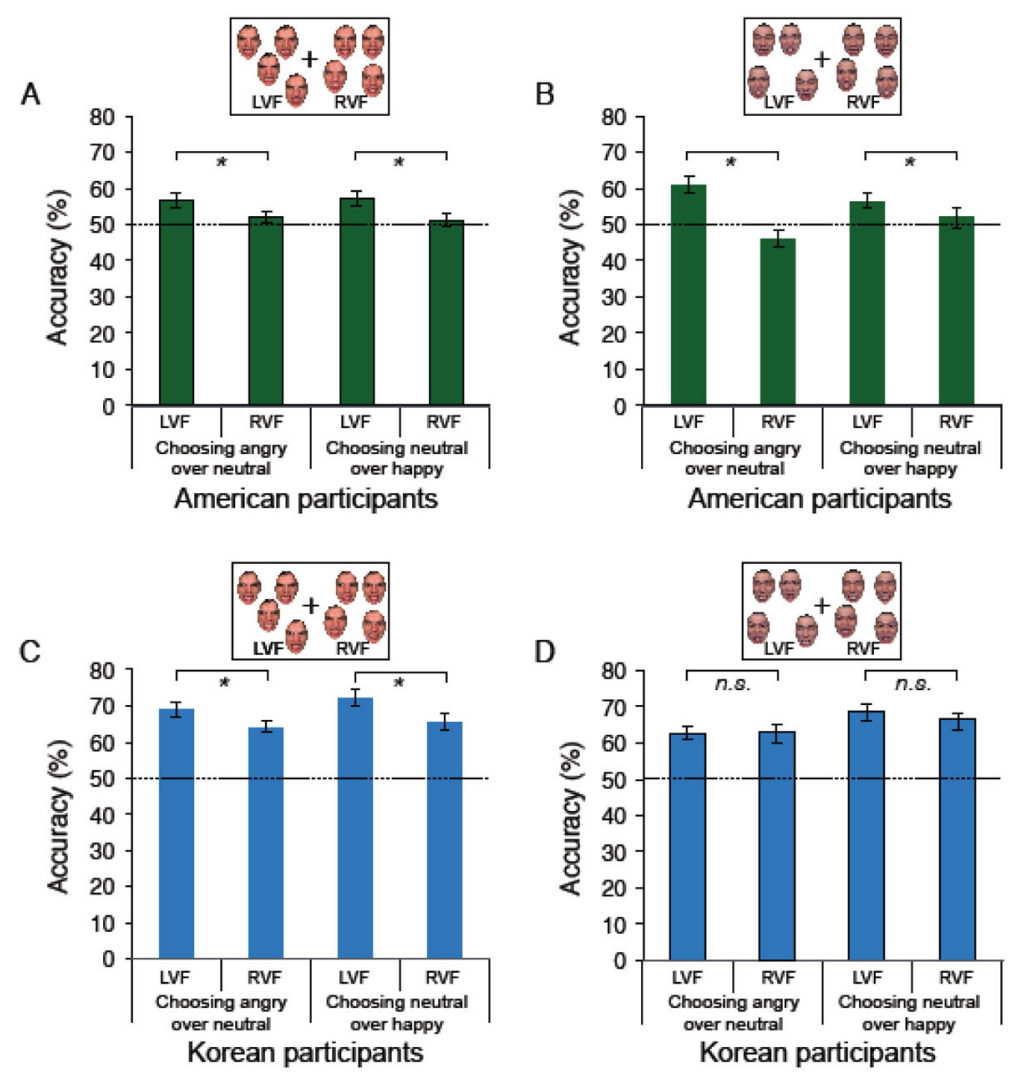

Figure 4. Laterality effect in Experiment 2

(A) The American participants' accuracy for American facial crowds when they had to choose an angrier and a neutral facial crowd presented in the LVF and the RVF. The broken line indicates the chance level, 50\%. The error bars indicate the standard error of the mean (SEM). (B) The American participants' accuracy for Korean facial crowds when they had to choose an angrier and a neutral facial crowd presented in the LVF and the RVF. (C) The Korean participants' accuracy for American facial crowds when they had to choose an angrier and a neutral facial crowd presented in the LVF and the RVF. (D) The Korean participants' accuracy for Korean facial crowds when they had to choose an angrier and a neutral facial crowd presented in the LVF and the RVF. 